\title{
Hacia una reflexión sobre la crisis ambiental. Max Horkheimer $y$ Günther Anders: Afán de dominio y desfase prometeico
}

Towards a reflection on the environmental crisis.

Max Horkheimer and Günther Anders: dominance desire and Promethean Discrepancy

Mtra. Gabriela Macedo Osorio

Universidad Nacional Autónoma de México gabriela.macedo.osorio84@gmail.com

DOI: http://doi.org/10.15366/bp2019.21.004 Bajo Palabra. II Época. No21. Pgs: 81-94 
El presente artículo fue realizado gracias al apoyo del proyecto de investigación en la UNAM PAPIIT IN 402317 "Heteronomías de la justicia: nomadismo y hospitalidad en el lenguaje".

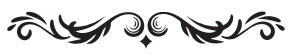

\section{Resumen}

La crisis ambiental que sufrimos incrementa en la medida en que el ser humano ha expandido los límites de aquello que considera, se encuentra bajo su escrutinio y dominio. Bajo la idea de progreso y con ello del trabajo científico, la naturaleza ha sido "desencantada" para volverse objeto de estudio, de análisis, experimentación y alteración con el objetivo, ya no de la búsqueda de una verdad en sí misma, sino de control, manipulación y transformación. El objetivo del texto es abordar los puntos de encuentro entre la crítica realizada por Max Horkheimer a las promesas incumplidas del proyecto ilustrado y del dominio que ejerce la razón instrumental sobre la naturaleza física y humana, con las críticas y advertencias que realiza Günther Anders sobre las consecuencias que genera la "vergüenza prometeica" y el "desfase prometeico" frente a la "ceguera ante el Apocalipsis".

Palabras Clave: crisis ambiental, dominio, antropología negativa, tecnología, responsabilidad.

\section{Abstract}

The environmental crisis that we suffer increases as far as the human being has expanded the limits of what he considers, is under his scrutiny and domination. Under both the idea of progress and of scientific work within it, nature has been "disenchanted" to become the object of study, analysis, experimentation and alteration, no longer with the objective, of searching for a truth in itself but to control it, manipulate it and transform it. The objective of the text is to address the points of convergence between, on one hand, the criticism made by Max Horkheimer on the unfulfilled promises of the enlightened project and the dominion exercised by instrumental reason over physical and human nature and, on the other, the criticisms and warnings made by Günther Anders concerning the consequences generated by the "Promethean shame" and the "promethean phase" on the "blindness before the Apocalypse".

Keywords: environmental crisis, dominion, negative anthropology, technology, responsibility. 
Hemos de abandonar definitivamente la esperanza ingenuamente optimista del siglo XIX de que las «luces» de los seres humanos se desarrollarían a la par que la técnica. Quien aún hoy se complace en tal esperanza no es sólo un supersticioso, no es sólo una reliquia de antaño, sino que es víctima de los actuales grupos de poder: de esos hombres oscuros de la época técnica cuyo máximo interés es mantenernos en la oscuridad en relación con la realidad del oscurecimiento de nuestro mundo o, mejor dicho, producir incesantemente esta oscuridad.

GüNTHer Anders, Nosotros los hijos de Eichmann ${ }^{1}$

E n las últimas décadas hemos vivido bajo la amenaza de nuestras creaciones, los efectos que creímos resultarían visibles "a largo plazo" no sólo nos han alcanzado, sino que han desbordado lo que se intentó predecir de ellos.

La contaminación atmosférica, de los suelos y del mar es muestra constante de los residuos que dejan tras de sí la producción industrial que ha realizado la humanidad, primero con el uso del carbón y posteriormente con el petróleo y sus derivados.

El Programa de las Naciones Unidas para el Medio Ambiente calcula que ocho millones de toneladas de plástico llegan al mar cada año, y de seguir así, se estima que para el 2050 los océanos tendrán más plástico que peces, y el 99\% de las aves marinas habrán ingerido plástico, porque en estos momentos el 60\% de estas mismas aves poseen ya este derivado del petróleo dentro de su sistema digestivo ${ }^{2}$.

El crecimiento demográfico en conjunto con la producción descomunal de bienes y servicios ha traído consigo una realidad de la que no todos aún son conscientes $^{3}$, pero que día a día todos sufrimos sus efectos; y es que resulta innegable que vivimos dentro de un mundo tecnológico, rodeados de artefactos que creamos

1 Anders, Günther, Nosotros los hijos de Eichmann, Barcelona, Paidós, 2001, p. 29.

2 Esta información fue brindada por Alejandro Laguna, oficial de Información del PNUMA, y publicada en la página de noticias de la ONU el 23 de febrero de 2017. También explicó que en los últimos 50 años se ha multiplicado veinte veces la producción mundial de plásticos, llegándose a generar 320 millones de toneladas en ese plazo. http://www.un.org/spanish/News/story.asp?NewsID=36831\#.WYZFuIWcH4g

${ }^{3}$ Existen grupos de científicos, entre ellos la Coalición Científica Internacional del Clima (ICSC por sus siglas en inglés) que afirman que el cambio climático no es causado por el ser humano, sino que lo atribuyen a cuestiones naturales, es decir: ciclos solares, erupciones volcánicas, movimientos en las corrientes marítimas, y no sólo a 
y desechamos diariamente, con los cuales elaboramos y reconfiguramos estrechas relaciones.

"Cuantos más aparatos inventamos para el dominio de la naturaleza, tanto más tenemos que ponernos a su servicio para sobrevivir" ", afirmó Max Horkheimer en 1947 en Critica de la razón instrumental buscando describir y explicar, desde su exilio en Estados Unidos, cómo es que el ser humano ha transformado a la naturaleza (cielo, tierra), en medio para su propia auto conservación; vaciándola de contenido, "desencantándola" diría siguiendo a Max Weber, "degradándola a mero material, a mera materia prima, que ha de ser dominada sin otro fin ni objetivo que el del dominio mismo"s.

Diecisiete años antes, en 1930, Günther Anders, afirmaba: "Como Homo Faber, el hombre hace algo del mundo, lo modifica con su intervención, le transmite su propio devenir; crea en él especies nuevas e imprevisibles, construye un mundo propio, una "superestructura". El hombre [...] necesita un mundo diferente para vivir, para superar, a través de la invención, el mundo que se le ofrece" ${ }^{6}$ Estas palabras formarían parte de una conferencia titulada "La extrañeza del hombre respecto al mundo", y posteriormente constituiría parte de un ensayo llamado: "Una interpretación del a posteriori”, la cual leería Anders, en la Kant-Gesellschat en la ciudad de Hamburgo y Frankfurt frente a dos miembros de la Escuela de Frankfurt: Theodor Adorno y Max Horkheimer; así como, el sociólogo Karl Mannheim, el teólogo Paul Tillich, el psicólogo Max Wertheimer y la filósofa Hannah Arendt, en ese momento, su esposa ${ }^{7}$.

Esta es la única ocasión en la que nuestros filósofos en cuestión entran en contacto, Max Horkheimer tiene en ese entonces 35 años, director del Instituto para Investigación Social y encargado de la cátedra de filosofía social en la Universidad de Frankfurt; y Günther Anders, de 28 años, egresado de la Universidad de Friburgo con una tesis doctoral dirigida por Edmund Husserl, con la intención de integrarse laboralmente al ámbito universitario. Ambos de padres judíos y con una cultura permeada por el cosmopolitismo alemán, realizarán un análisis y una crítica social desde su carácter de judíos exiliados en Estados Unidos, a causa de la persecución nazi en Europa. Peter Gay los describirá como "marginales del interior" (outsider as

\footnotetext{
la acción humana. https://www.elconfidencial.com/tecnologia/2015-11-30/siete-argumentos-de-los-negacionistas-del-cambio-climatico-y-sus-respuestas-cientificas_1109578/

${ }^{4}$ Horkheimer, Max, Crítica de la razón instrumental, Madrid, Trotta, 2002, p. 119.

5 Ídem.

${ }^{6}$ Anders, Günther, "Una interpretación del a posteriori” en Acerca de la libertad, Valencia, Pre-Textos, 2014, p. 53.

7 Cfr. Maomed Parraguez y De Vicente Hernando, César, “El tiempo de la antropología filosófica” en Anders, Günther, Acerca de la Libertad, Valencia, Pre-Textos, 2014.
} 
insiders), que encontrarán su "verdadero hogar" en el exilio. ${ }^{8}$ Sus análisis, descripción y crítica de lo vivido muestra, en nuestros autores, una desilusión, pesimismo y crítica frente a las promesas planteadas por un sistema moderno que invitaba a preponderar el uso de la razón como fuente de conocimiento y legitimación. Una etapa de progreso que no presentaba más que dominio e instrumentalización sobre el otro.

Esta mirada "de los de fuera" que irrumpe el continuum de la historia, invita a detenerse y observar lo que pareciera estar ya dado por hecho, es una especie de "privilegio epistemológico del exilio", tal como afirma Enzo Traverso.

Esta mirada algo desfasada, puede volverse una ventaja: permite ver lo que otros no ven. [...] Por la mirada a la vez interna y externa que tiene de la sociedad, el extranjero está mejor posicionado para tener una visión crítica y anticonformista, que escapa a las convenciones y a las ideas aceptadas. [...] el exilio crea la condición de esa mirada otra que puede ser particularmente penetrante. Durante la Segunda Guerra Mundial, sobre todo son los exiliados quienes vieron y pensaron Auschwitz. ${ }^{9}$

Los puntos de intersección que mantienen sus investigaciones a lo largo de su vida, da muestra, dan muestra no sólo de la preocupación de la época, sino también del alcance que llegarán a tener sus pensamientos y estudios en el análisis filosófico de la problemática ambiental; caso específico de nuestra investigación. Por todo lo cual, el objetivo de mi ponencia es esbozar algunos puntos de encuentro entre la crítica realizada por Max Horkheimer a las promesas incumplidas del proyecto ilustrado y del dominio que ejerce la razón instrumental sobre la naturaleza física y humana, con las críticas y advertencias que realiza Günther Anders bajo la reacción que genera la "vergüenza prometeica" y el "desfase prometeico" frente a la "ceguera ante el Apocalipsis".

Dos preguntas guiarán nuestro escrito: ¿Cuál es el origen de esta relación que mantiene el hombre con la naturaleza? ¿Cuáles son las consecuencias que devienen de esta relación?

Ambos filósofos analizan la relación entre el ser humano, la ciencia y la naturaleza pero bajo enfoques e intereses distintos mas no por eso incompatibles, pues mientras el enfoque de Max Horkheimer dentro de la Escuela de Frankfurt tiende a un análisis y una crítica social y política sobre las implicaciones de la tecnología; la de Günther Anders, no inscrito bajo ninguna universidad o grupo académico,

\footnotetext{
${ }^{8}$ Peter Gay citado por Traverso, Enzo, Cosmópolis. Figuras del exilio judeo-alemán, México, Universidad Nacional Autónoma de México/Fundación Eduardo Cohen, 2004, p. 43.

9 Ibíd., p. 9-10.
} 
busca dilucidar, criticar y denunciar el peligro latente que afecta a la humanidad respecto a su quehacer frente al uso de la tecnología, a partir de su propuesta de "antropológica negativa".

Mientras que para Max Horkheimer la relación que mantiene el ser humano con la naturaleza está basada en el dominio adscrito a la preponderancia, cada vez mayor, de la razón instrumental plasmada en la ciencia positiva; en el caso de Günther Anders, la relación del hombre con la naturaleza también es una relación de dominio, pero que parte del temor y la vergüenza por la condición contingente y de extrañeza de ser humano, de su cuerpo y condición mortal e inacabada frente al entorno que le rodea.

Günther Anders describe el mundo tecnológico como un "macro aparato" que no sólo interpreta la forma en que el ser humano ha creado una segunda naturaleza para poder adaptarse a las inclemencias de la naturaleza, sino que, con el pasar de los años, ha generado cuestionamientos sobre la auto comprensión del propio ser humano y su destino.

Y es que, para el autor de la Obsolescencia del hombre, el problema central del mundo tecnológico es la discrepancia existente entre el enorme poder tecnológico que el ser humano ha creado y las capacidades limitadas que parece tener, para comprender, representar y controlar sus efectos. Es decir, podemos hacer mucho, individual y colectivamente, pero nuestra conciencia se ha desfasado respecto a lo que podemos hacer y provocar con nuestras creaciones y actos. A esta condición, le llamó Anders, el desfase prometeico (das prometheische Gefälle). Bajo esta condición, "[...] la transformación de los aparatos avanza absolutamente con demasiada rapidez; que los productos nos exigen algo exagerado, algo imposible; y que con su exigencia nos colocan realmente en una situación patológica colectiva." 10

Todo ello, resulta preocupante, ya que la conciencia humana no está transformándose para poder comprender los cambios que generan las revoluciones tecnológicas, porque el ser humano no alcanza a comprender la enorme dimensión y responsabilidad que sus creaciones, acciones y actos tienen, ahora que están inscritos o son originados por la tecnología.

[...] desde la perspectiva del productor: no resultaría imposible que nosotros, que producimos estos productos, estemos a punto de establecer un mundo, cuyo paso no somos capaces de seguir, y "aprehenderlo" supera absolutamente nuestra capacidad de comprender, la capacidad tanto de nuestra fantasía como de nuestras emociones. Quién sabe, tal vez hayamos establecido ya un mundo así. ${ }^{11}$

\footnotetext{
${ }^{10}$ Anders, Günther. La Obsolescencia del hombre. Sobre el alma en la época de la segunda revolución industrial. Vol. 1, Valencia, PRETEXTOS, 2011 p. 32-33.

${ }^{11}$ Ibíd., p.33.
} 
Pero ¿cómo es la relación que mantiene el ser humano con este mundo tecnologizado? ¿qué clase de ser humano ha conformado? La respuesta a estos cuestionamientos la plantea Anders a partir una antropología negativa, en ella explica que el ser humano se percibe con un ser ajeno y extraño en el "mundo natural"; observa que su cuerpo no tiene las habilidades que poseen otros animales para desarrollarse y vivir en la naturaleza; es por ello que busca transformar su entorno, adaptarlo a sus necesidades; para ello, crea herramientas, utensilios, maquinaria que le permita "forjarse" una segunda naturaleza.

El optimismo prometeico respecto a que todo, no sólo se podía modificar y transformar por y a favor del ser humano, sino que se debia hacer, llevó a nuestro autor a considerar el advenimiento de una nueva era, la que traería consigo la Tercera Revolución Industrial caracterizada por el uso de la tecnología; pues con el pasar del tiempo, las herramientas se hicieron cada vez más sofisticadas, y la tecnología dio pauta para que el impulso del Homo Creador pudiera transformar todas las cosas que le rodeaban de un modo más eficiente, pero también, más dominante y sustancial.

Para el filósofo de Bresau, la naturaleza contingente y asincrónica del ser humano lo hacía, por un lado, percibirse como un ser exiliado del mundo, es decir, extraño y ajeno ante una realidad que se muestra hostil frente a su presencia contingente y que le hace cuestionarse sobre su lugar y propósito en el mundo; y a consecuencia de ello mantiene una relación "discrepante", es decir desnivelada entre su producir (herstellen) y su imaginar (vorstellen). $\mathrm{Y}$ es que, si el ser humano no encuentra un lugar vinculante dentro del mundo natural, su relación con la naturaleza se basará en la transformación de un lugar en el mundo a partir de las habilidades y capacidades que tiene, siendo la capacidad racional, la más distinguible y característica. A partir de ella buscará explicar, pero también dominar-controlar y someter, aquello que desconoce y le causa temor; orientarlo, transformarlo y recrearlo para hacerlo más provechoso y útil a sus fines.

$\mathrm{Y}$ es bajo esta última afirmación que converge uno de los objetivos principales Dialéctica de la Ilustración, esto es, la explicación sobre el proceso que llevó a que los ideales de la Ilustración, de la idea de progreso, de la confianza que se tenía en la razón para realizar un proyecto en común, derivaran en la exclusión, la muerte burocratizada y el dominio de la razón instrumental.

Así, explica Horkheimer en Dialéctica de la Ilustración, el objetivo era liberar a los hombres del miedo y constituirlos en señores ${ }^{12}$ disolviendo los mitos y derrocando la magia por medio de la actividad científica; de esta forma, se concibe la

${ }^{12}$ Cfr. Horkheimer y Adorno, Dialéctica de la Ilustración, Madrid, Trotta, 2006, p.59. 
estructura de la razón ilustrada como aquella que por medio del saber se apodera del mundo, haciéndolo propio en el sentido de establecer límites conceptuales, eliminando poco a poco ambigüedades metafísicas y estructurando la realidad a partir de considerar a ésta como la única fuente de conocimiento y de acercamiento al lenguaje de la naturaleza.

Pero la paradoja interna que posee la Ilustración en la que, por un lado, desea librarse de los mitos imponiendo la luz de la racionalidad configura un nuevo mito que justifica el poder de la razón, y que sobre todo nos muestra cómo la naturaleza dialéctica de la razón (objetiva y subjetiva) llevará al desequilibrio que resulta en razón instrumental. Pues que el mito sea ya Ilustración y la ilustración recaiga en mitología nos revela las dos caras de una misma moneda, su naturaleza dialéctica.

La Ilustración, afirman, se esfuerza por desmitificar a la naturaleza operando sobre el mismo mito que busca eliminar. "La aporía ante la que nos encontramos en nuestro trabajo se reveló, así como el primer objeto que debíamos analizar: la autodestrucción de la Ilustración. [...] la causa de la regresión de la Ilustración a mitología no debe ser buscada tanto en las modernas mitologías nacionalistas, paganas y similares, [...] sino en la Ilustración misma paralizada por el miedo a la verdad" 13 .

Para Horkheimer, la razón posee en sí misma una estructura dialéctica orientada por una lógica de dominio que la impulsa a conocer, no sólo para adquirir conocimiento por sí misma, sino para dominar y controlar lo externo; aquello que es distinto, diferente, misterioso. De esta forma la Ilustración, al ser tomada como el periodo que representa este auge de conocimiento y descubrimiento, es conducida también bajo el signo del dominio.

Para Horkheimer y Adorno es importante destacar que el carácter de dominio de la razón, propio de la razón instrumental, no es una enfermedad de la razón, porque esto indicaría que la razón, a lo largo de la historia estuvo "sana" y "algo" la atacó contagiándola; al contrario, para los autores de la Dialéctica de la Ilustración el afán de dominio es una parte inseparable de la esencia de la razón que explica el desarrollo de la humanidad, pues si bien la crisis surgió en la Modernidad, no significa que no haya estado antes presente, sino que las condiciones de la Ilustración favorecieron su expresión a gran escala.

De hablarse de una enfermedad que afecta a la razón, ésta no debería ser entendida en el sentido de haber afectado a la razón en un momento histórico determinado, sino como inseparable de la esencia de la razón en la civilización, tal como la hemos conocido hasta la fecha. La enfermedad de la razón tiene sus raíces en su origen, en el afán del hombre de dominar la

13 Ibíd. p. 54. 
naturaleza [...] Desde la época en que la razón se convirtió en el instrumento del dominio de la naturaleza humana y extra humana por el hombre - esto es, desde los más tempranos comienzos-, su intención propia, la de descubrir la verdad, se ha visto frustrada. Lo cual debe atribuirse a su conversión de la naturaleza a mero objeto, y al hecho de haber fracasado en el empeño de descubrir la huella de sí misma en tal objetivación, en los conceptos de materia y de cosa no menos que en los de los dioses y del espíritu. ${ }^{14}$

De esta forma la voluntad de dominio, definida por Horkheimer como "la necesidad social de controlar la naturaleza ha condicionado siempre la estructura y las formas del pensamiento humano, confiriendo el primado a la razón subjetiva" 15

El carácter de dominio que presenta como característica la razón instrumental contrasta con las promesas de progreso y beneficio que aseguraba la Modernidad, puede si bien "ser modernos es encontrarnos en un entorno que nos promueve aventuras, poder, alegría, crecimiento, transformación de nosotros y del mundo [...] al mismo tiempo amenaza con destruir todo lo que tenemos, todo lo que sabemos, todo lo que somos" ${ }^{16}$.

La naturaleza es enfatizada como un objeto ajeno al hombre, puesto a su disposición, al mismo tiempo que los hombres se esfuerzan por sobrevivir dentro de un medio que, aunque creado por ellos, les exige relativizar los fines y llevar a identificar la razón con la capacidad de adaptación.

Las fuerzas económicas y sociales asumen el carácter de potencias naturales ciegas que el hombre ha de dominar, adaptándose a ellas para sobrevivir. Como resultado final del proceso tenemos por una parte el sí mismo, el yo abstracto, vaciado de toda substancia que no sea su intento de convertirlo todo, en el cielo y en la tierra, en un medio para su conservación y prevalecimiento; y, por otro, tenemos una naturaleza vacía, degradada a mero material, a mera materia primera, que ha de ser dominada sin otro fin ni objetivo que el del dominio mismo ${ }^{17}$.

Para Horkheimer, la relación que se establece entre el hombre y la naturaleza, mediada por la ciencia, es semejante a la de un dictador con los hombres, ya que "[...] Éste los conoce en la medida en que puede manipularlos. [Asi pues] El hombre de la ciencia conoce las cosas en la medida en que puede hacerlas." $18 \mathrm{Y}$ es que, si bien la crítica que realiza Horkheimer a la ciencia es pensando en la ciencia positiva,

\footnotetext{
14 Ibíd., p. 179.

15 Horkheimer, Max, Critica a la razón instrumental, op. cit., p.179.

${ }^{16}$ Arriarán, Samuel, Filosofía de la posmodernidad. Crítica a la modernidad desde América Latina, México, Facultad de Filosofía y Letras, Universidad Nacional Autónoma de México, 1997, p. 155.

17 Horkheimer y Adorno, Crítica a la razón instrumental, op. cit., p.119.

18 Ibíd., p.64.
} 
Anders se dirige al quehacer de la tecnología, en concreto, al trabajo fraccionado en el que no sólo la industria, sino también los laboratorios científicos de hoy en día, realizan investigaciones y experimentaciones bajo un carácter "colaboracionista", fraccionado, tecnificado, en el cual da lo mismo lo qué se produzca, pues al repartirse en tantas partes la labor, no es posible percibir los fines ni las consecuencias del trabajo en conjunto; además, advierte, no hay un responsable, pues cada individuo al trabajar de manera dividida, sólo podría responder, en el mejor de los casos, de aquella sección que realizó y no de la acción técnica a gran escala y, mucho menos, de las repercusiones futuras que conllevara su proyecto.

En este punto podríamos preguntarnos ¿cómo la humanidad continua? ¿por qué no se ha hecho nada al respecto, alguna acción tajante que pueda disminuir o contener los actos que nos llevan, afirmaría Anders, de camino al Apocalipsis? En la respuesta confluyen nuevamente nuestros autores en afirmar que el progreso es un factor relevante para esta ceguera. Pues para el autor de la Crítica a la Razón Instrumental, la idea de "progreso" humano tiene su fundamento en la naturaleza dialéctica de la razón, y con ello, en la predominancia del carácter subjetivo de la razón, que se torna razón instrumental, ya que dicha barbarie no es resultado de fuerzas degeneradas, excepcionales o irracionales que "aparecen" de manera extraña en el curso de la historia; todo lo contrario, la violencia que se reproduce, de distintas formas, tiene que ver con los procesos de emancipación que han surgido de la sociedad moderna.

Pero paradójicamente, el énfasis excesivo en la autonomía humana, en el poder de la razón sobre todas las cosas, fue lo que llevó a la propia sumisión de los hombres, cuando la misma idea de progreso, transformación y dominio se convirtió en el destino propio del hombre, pues una vez [...] llevada a su extremo lógico, la racionalidad formal, instrumental y calculadora, conducía a los horrores de la barbarie del siglo XX. ${ }^{19}$

Y si ligamos la relación que mantiene este carácter de dominio de la razón instrumental con la ciencia positiva, es posible afirmar el poder de la ciencia como principio regulador de la sociedad. La ciencia suministra un dominio sobre la naturaleza ya que, frente al desenvolvimiento de la técnica y la experimentación, el progreso de la humanidad se radica en un uso meramente instrumental, es decir en su manipulación y explotación.

El universo entero es convertido en un instrumento del yo, por mucho que éste no tenga substancia o significado alguno fuera de su propia actividad ilimitada, [...] la naturaleza es considerada hoy más que nunca como un mero instrumento de los hombres. Es el objeto

19 Jay, Martin, La Imaginación dialéctica, Madrid, Taurus, 1989, p.427. 
de una explotación total que no conoce objetivo alguno puesto por la razón y, por lo tanto, ningún límite. El imperialismo de la especie humana sobre la tierra no tiene paralelo alguno en aquellas épocas de la historia natural en las que otras especies animales representaban las formas más altas de evolución orgánica. ${ }^{20}$

En el caso de Günther Anders, la "ceguera ante el Apocalipsis" (Apokalypse-Blindheit) está sustentada en dos causas entrelazadas, la primera es la ilusión ilustrada del progreso, esto es, en una sociedad tecnológica en la que el futuro ya no es objeto de una espera incierta de designios divinos ni de fuerzas desconocidas, sino que el ser humano tiene la capacidad no sólo de anticiparse o predecir acontecimientos naturales, como lo pudieran ser las tormentas, temblores, eclipses; ya que ahora puede producirlos. El futuro ya no acontece, sino que se produce según intereses económicos y políticos.

La segunda causa radica en el desfase prometeico, la cual consiste en la poca o nula habilidad por comprender e imaginar las terribles consecuencias que devienen de las creaciones. Pues vivimos bajo el influjo de una fascinación por el poder tecnológico que "hoy por hoy constituyen la fuente de un riesgo mayor para el futuro de la condición humana, e incluso para su mera supervivencia" ${ }^{21}$. Ya que nosotros, los seres humanos, herederos del moderno Prometeo, los primeros en dominar el Apocalipsis, también seremos los primeros en perecer por nuestra falta de responsabilidad, conciencia e imaginación; pues incluso hemos llegado a la creación y utilización de armamentos de destrucción masiva que han convertido a la humanidad entera en la especie más vulnerable y aniquilable por sí misma.

Si en la conciencia del hombre actual hay algo que se considera absoluto o infinito, ya no es el poder de Dios, tampoco el poder de la naturaleza, por no hablar de los supuestos poderes de la moral o la cultura, sino nuestro poder. En lugar de la creatio ex nibilo, que manifiesta omnipotencia, ha aparecido su contrapoder: la potestas annibilationis, la reductio ad nibil; un poder que está en nuestra propia mano. La omnipotencia prometeica, anhelada desde tanto tiempo, se ha hecho en verdad nuestra, aunque de forma diferente a la esperada. Dado que poseemos el poder de ponernos fin unos a otros, somos los señores del apocalipsis. Lo infinito somos nosotros. 22

Pero qué alternativa nos queda sino la de ser conscientes de nuestras incapacidades, deficiencias y responsabilidades, al comprometernos con nuestras acciones;

\footnotetext{
${ }^{20}$ Horkheimer y Adorno, Crítica a la razón instrumental, op. cit., p.127.

21 Linares, Jorge Enrique, Ética y mundo tecnológico, México, Fondo de Cultura Económica/Universidad Nacional Autónoma de México, 2008, p. 193.

22 Anders, La obsolescencia del ser humano, op.cit., p.230-231.
} 
convirtiendo tanto el presente como el futuro en objeto de nuestra responsabilidad $\mathrm{Y}$ es que, si somos capaces de gestar el porvenir, podemos también no ser ciegos ante los peligros que hemos suscitado. Hay que ampliar nuestra capacidad imaginativa para observar y actuar desde un horizonte más dilatado, e incluso exagerado, el alcance de nuestras acciones.

[...] se trata de "ampliar" una facultad [Vermögen]. Esta vez nuestra facultad para comprender el tiempo [Zeit-Vermögen]. Pero lo que se nos exige no es algo similar a lo profético para prever esto o lo otro, sino sólo que intentemos comprender el horizonte ampliad como nuestro, tal como lo hacemos desde un pico montañoso o desde un avión. [...] nosotros tenemos que captar los futuros acontecimientos, temporalmente más lejanos para sincronizarlos con nuestro único punto temporal, el ahora, como si sucedieran justamente en el presente. Pues acontecen hoy porque dependen del presente; $y$ en tanto acontecen actualmente, nos conciernen porque justo ahora los "solicitamos" a través de lo que hacemos en el presente. ${ }^{23}$

${ }^{23}$ Ibíd., p. 283-284. 


\section{RefERenCias Bibliográficas}

Anders, Günther. La Obsolescencia del hombre. Sobre el alma en la época de la segunda revolución industrial. Vol. 1, Valencia, PRETEXTOS, 2011.

- Nosotros los hijos de Eichmann, Barcelona, Paidós, 2001.

- "Una interpretación del a posteriori" en Acerca de la libertad, Valencia, Pre-Textos, 2014.

Arriarán, Samuel, Filosofía de la posmodernidad. Crítica a la modernidad desde América Latina, México, Facultad de Filosofía y Letras, Universidad Nacional Autónoma de México, 1997.

Horkheimer y Adorno, Dialéctica de la Ilustración, Madrid, Trotta, 2006.

Horkheimer, Max, Critica de la razón instrumental, Madrid, Trotta, 2002.

JaY, Martin, La Imaginación dialéctica, Madrid, Taurus, 1989.

Linares, Jorge Enrique, Ética y mundo tecnológico, México, Fondo de Cultura Económica/Universidad Nacional Autónoma de México, 2008.

Maomed Parraguez y De Vicente Hernando, César, "El tiempo de la antropología filosófica" en Anders, Günther, Acerca de la Libertad, Valencia, Pre-Textos, 2014.

Traverso, Enzo, Cosmópolis. Figuras del exilio judeo-alemán, México, Universidad Nacional Autónoma de México/Fundación Eduardo Cohen, 2004.

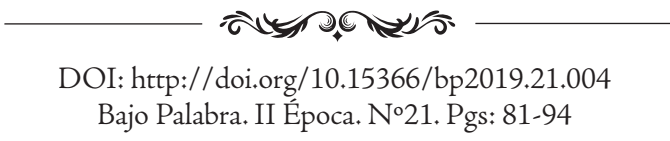


